\title{
Diversidade de abelhas (Hymenoptera: Apoidea) em uma área de transição Cerrado-Amazônia ${ }^{1}$.
}

\author{
Florisvaldo Mesquita dos SANTOS ${ }^{2}$, Carlos Alfredo Lopes de CARVALHO ${ }^{3}$, Rejane Ferreira SILVA ${ }^{4}$
}

\begin{abstract}
RESUMO
A comunidade de abelhas em uma área de transição cerrado-amazônia, localizada na região do Bico-do-Papagaio, Estado do Tocantins, foi estudada entre novembro de 1999 e novembro de 2000, por meio da amostragem sistemática dos espécimes nas plantas em floração, com o objetivo de obter informações sobre a sua composição. Um total de 5.534 indivíduos, distribuídos em 83 espécies e 38 gêneros foram coletados. A maior abundância de indivíduos e riqueza de espécies foi encontrado na família Apidae sensu Roig-Alsina \& Michener. Os gêneros com maior riqueza foram Trigona (8), Megachile (7), Centris (6), Augochloropsis (5), Coelioxys (5), Paratetrapedia (5) e Xylocopa (5), enquanto que a espécie mais freqüente foi Apis mellifera. A comunidade seguiu o padrão geral encontrado nos neotrópicos, apresentando muitas espécies com poucos indivíduos e poucas espécies com muitos indivíduos. A freqüência, constância e dominância das espécies são discutidas e os índices de abundância, diversidade, eqüitabilidade e dominância são apresentados.
\end{abstract}

\section{PALAVRAS-CHAVE}

Comunidade, Apidae, região do Bico-do-Papagaio.

\section{Diversity of bees (Hymenoptera: Apoidea) in transition area of Cerrado-Amazônia.}

\begin{abstract}
The bee community in a transition area of Cerrado-Amazônia in Bico-do-Papagaio region, in the of State of Tocantins, Brazil, was studied monthly from November, 1999 to November, 2000, by means of systematic sampling of bees in flowering plants, with the objective of obtaining information on its fauna composition. A total of 5534 individuals distributed in 83 species and 38 genera were collected. The largest abundance of individuals and richness of species was found in the family Apidae sensu Roig-Alsina \& Michener. The genera with largest richness of species were Trigona (8), Megachile (7), Centris (6), Augochloropsis (5), Coelioxys (5), Paratetrapedia (5), and Xylocopa (5), while the most frequent species was Apis mellifera. The community structure followed the general pattern found in neotropical areas, showing many species with few individuals and few species with many individuals. The frequency, constancy, and dominancy of species are discussed, and the ecological indexes are presented.
\end{abstract}

\section{KEY WORDS \\ Community, Apidae, Bico-do-Papagaio region.}

\section{INTRODUÇÃO}

As abelhas constituem os agentes polinizadores mais adaptados à visita das flores das Angiospermas. Suas relações baseiam-se em um sistema de dependência recíproca, onde as plantas fornecem o alimento para as abelhas, principalmente pólen e néctar, e em troca recebem os benefícios da transferência de pólen (Kevan \& Baker, 1983; Proctor et al., 1996).
O processo de polinização constitui uma das mais fortes ligações entre plantas e animais, geralmente para ambas as partes. O fluxo gênico entre membros de uma população vegetal depende do raio de alcance do polinizador, distribuição espacial da planta e da biologia floral (Barth, 1991).

A maior eficiência das abelhas como polinizadores se dá, tanto pelo seu número na natureza, quanto por sua melhor adaptação às complexas estruturas florais como, por exemplo, peças bucais e corpos adaptados para embeber o

\footnotetext{
${ }^{1}$ Parte da Dissertação de Mestrado apresentada ao Programa de Pós-Graduação em Ciências Agrárias da UFBA pelo primeiro autor.

${ }^{2}$ Universidade Federal da Bahia, Escola de Agronomia, Programa de Pós-Graduação em Ciências Agrárias, 44380-000. Cruz das Almas-BA.

${ }^{3}$ Prof ${ }^{\circ}$ Adjunto - Universidade Federal da Bahia, Escola de Agronomia, 44380-000. Cruz das Almas-BA. E-mail: calfredo@ufba.br

${ }^{4}$ Bióloga, Instituto de Desenvolvimento Rural do Estado do Tocantins, AANE 40, Lotes 1 e 2, Centro, 77.000-000, Palmas-TO.
} 


\section{ACTA \\ AMAZONICA}

DIVERSIDADE DE ABELHAS (HYMENOPTERA: APOIDEA)

EM UMA ÁREA DE TRANSIÇÃO CERRADO-AMAZÔNIA néctar das flores e coletar pólen, respectivamente (Kevan \& Baker, 1983; Proctor et al., 1996).

Estima-se que existam mais de quatro mil gêneros e cerca de 25 a 30 mil espécies distribuídas nas diferentes regiões do mundo (Michener, 2000). Cerca de $85 \%$ das espécies de abelhas descritas são solitárias (Batra, 1984), sendo que muitas dessas espécies pertencem à família Apidae (Roig-Alsina \& Michener, 1993). Representantes desta família podem voar longas distâncias nas matas tropicais em busca de espécies vegetais preferenciais, promovendo a polinização cruzada (Frankie et al. 1983; Roubik 1993).

O levantamento e a identificação das espécies de abelhas constituem no primeiro passo para se conhecer os polinizadores e definir estratégias de exploração racional e conservação dos recursos biológicos encontrados nas comunidades de vegetais e de animais (Kevan \& Baker, 1983; Matheson et al., 1996; Proctor et al., 1996).

A região do Bico-do-Papagaio, Estado do Tocantins, é considerada uma zona de grande diversidade ambiental e biológica e, também, é uma área de alta pressão ecológica e tensão social, com forte ocupação humana.

Desta forma, este trabalho teve como objetivo contribuir para o conhecimento da diversidade de abelhas em uma área de transição cerrado-amazônia sob fortes impactos ambientais localizada na região do Bico-do-Papagaio, norte do Estado do Tocantins.

\section{MATERIAL E MÉTODOS}

Duas trilhas de coleta com aproximadamente 1000 metros de extensão, foram demarcadas nas localidades de Esperantina (5 $20^{\prime} 16^{\prime \prime} \mathrm{S}$; $48^{\circ} 35^{\prime} 28^{\prime \prime}$ W; Altitude: $118,88 \mathrm{~m}$ ) e Mulatos ( $5^{\circ} 20^{\prime} 28^{\prime \prime} \mathrm{S}$; $48^{\circ} 29^{\prime} 12^{\prime \prime}$ W; Altitude: 144,94m), município de Esperantina-TO, região do Bicodo-Papagaio.

O período de coleta foi entre 30/11/1999 e 08/11/2000. $\mathrm{O}$ material coletado foi encaminhado para o Laboratório de Entomologia da Escola de Agronomia/UFBA para ser triado, identificado e quantificado.

O regime de chuvas na região do Bico-do-Papagaio segue o padrão tropical, com dois principais períodos no ano: chuvoso, que se inicia em dezembro e se prolonga até abril, e seco com precipitações esparsas, de maio a novembro. A precipitação pluviométrica média mensal é de $121,75 \mathrm{~mm}$ e a temperatura média anual é de $26,49^{\circ} \mathrm{C}$. A variação mensal das chuvas no período foi obtida na Estação da CPRM em Araguatins-TO, localizada, aproximadamente, a $60 \mathrm{Km}$ das trilhas de coleta (Figura 1).

A vegetação, originalmente formada por cerrado e floresta ombrófila, encontra-se bastante alterada, sendo resultado do desmatamento para implantação de pastagem e outras atividades agrícolas, constituindo-se principalmente de espécies arbustivas e herbáceas com elementos de vegetação ruderal.

\section{Amostragem}

As coletas das abelhas foram realizadas quinzenalmente por meio de redes entomológicas ao longo das duas trilhas. Para cada trilha de amostragem foi formada uma dupla de coletores e outra de auxiliares, que procederam à coleta das abelhas nas flores e observações gerais no dia da coleta. Os coletores e seus auxiliares partiram de pontos opostos da trilha de coleta, cobrindo as diferentes posições ao longo do dia.

A metodologia de coleta das abelhas foi baseada em Sakagami et al. (1967), cujos dados obtidos representam, de forma satisfatória, a estrutura faunística das áreas estudadas (Barbola \& Laroca, 1993; Aguiar et al., 1995; Carvalho, 1999). Os coletores se deslocaram na trilha das 5:00 às 18:00 horas, capturando qualquer abelha que estivesse visitando flores. Esse percurso foi realizado duas vezes durante as coletas (manhã e tarde), onde foram gastos, aproximadamente, entre 5 a 10 minutos por planta.

As abelhas coletadas foram mortas com acetato de etila embebido em algodão, previamente colocado em diferentes recipientes. A identificação foi baseada nos exemplares depositados no Museu Entomológico da Escola de Agronomia da Universidade Federal da Bahia e chaves taxonômicas. Algumas espécies foram encaminhadas para especialistas. Os exemplares foram depositados na Coleção de Abelhas do Instituto de Desenvolvimento Rural do Estado do Tocantins (Ruraltins) em Palmas-TO, sendo que, duplicatas foram doadas ao Museu de Entomologia da Escola de Agronomia da UFBA e aos especialistas que colaboraram com a identificação das espécies.

\section{Análise da Comunidade}

A eficiência do número de coletas na representatividade das espécies que ocorrem na área de estudo foi avaliada através da Curva do Coletor conforme Colwell \& Coddington (1994). A validação estatística para o modelo encontrado foi por meio da análise de regressão conforme Carvalho (1999).

A comunidade de abelhas foi caracterizada por meio

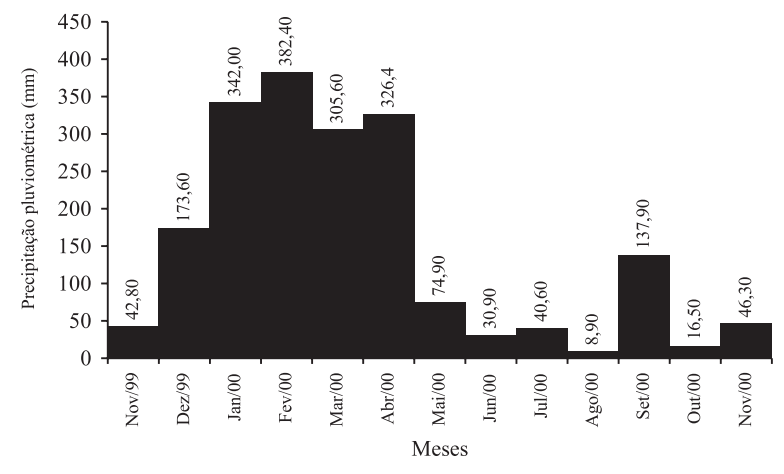

Figura 1 - Precipitação pluviométrica na Estação da CPRM em Araguatins-TO, região do Bico-do-Papagaio: 1999-2000. 
de diferentes índices faunísticos: freqüência relativa e constância (Silveira Neto et al., 1976), dominância das espécies (Kato et al., 1952), índice a (Margalef), abundância $S$, diversidade $H^{\prime}$ (Shannon-Wiener) e $\lambda$ (Simpson) (Ludwig \& Reynolds, 1988) e eqüitabilidade (J') Pielou (1977).

Foram estabelecidas classes de freqüência para cada espécie, por meio de Intervalos de Confiança (IC) a 5\% de probabilidade: pouco freqüente $(\mathrm{PF})=f<$ que o limite inferior (LI) do $\mathrm{IC}_{5 \%}$; freqüente $(\mathrm{F})=f$ situado dentro do $\mathrm{IC}_{5 \%}$; muito freqüente $(\mathrm{MF})=f>0$ limite superior (LS) do $\mathrm{IC}_{5 \%}$.

Para a constância das espécies nas coletas utilizou-se a classificação de Bödenheimer (citado por Silveira-Neto et al., 1976), sendo consideradas como espécies constantes (W) quando a porcentagem de ocorrência for igual ou superior a $50 \%$; espécies acessórias $(\mathrm{Y})$, quando a porcentagem de ocorrência for entre 25 e 50 \%; e espécies acidentais (Z), quando a porcentagem de ocorrência for igual ou inferior a $25 \%$.

A dominância (D) de uma determinada espécie foi identificada, quando o limite inferior do seu intervalo de confiança (Kato et al., 1952) foi maior que o inverso do número total de espécies multiplicado por 100 , conforme Sakagami \& Matsumura (1967). Esse mesmo procedimento também foi utilizado por diversos autores como Cure et al. (1993), Aguiar \& Martins (1997) e Carvalho (1999).

\section{RESULTADOS E DISCUSSÃO}

A comunidade de abelhas foi representada por 83 espécies (abundância S), 38 gêneros, 15 tribos, sete subfamílias e cinco famílias, com um total de 5.534 indivíduos coletados (Tabela 1).

A maior riqueza de espécies (Figura 2) e abundância de indivíduos foi encontrada na família Apidae $(68,67$ \% e $97,20 \%$, respectivamente)

Ao nível de família, a riqueza de espécies nas áreas tropical e subtropical da região Neotropical apresenta uma tendência de ser maior em Anthophoridae (sensu stricto), Megachilidae e Halictidae, enquanto que nas áreas temperada e subtropical dessa mesma região, a maior riqueza é observada em Halictidae, Anthophoridae (sensu stricto) e Megachilidae. Levantamentos em diferentes áreas demonstraram que Anthophoridae (sensu stricto) possui elevada proporção de espécies nas diferentes latitudes; Halictidae aumenta em abundância nas maiores latitudes do Sul do Brasil e Megachilidae possui proporção variada de espécies nas diferentes regiões (Sakagami \& Matsumura, 1967; Sakagami et al., 1967; Heithaus, 1979; Roubik, 1989; Wittmann \& Hoffman, 1990; Carvalho, 1999).

A família Apidae sensu stricto (tribo Apini e subtribos Apina, Bombina, Euglossina e Meliponina) aumenta em riqueza de espécies na direção das baixas latitudes, enquanto que, Andrenidae e Colletidae são pouco representadas na região Neotropical (Bortoli \& Laroca, 1990; Martins, 1994; Silveira \& Campos, 1995).

O sucesso de Apidae nas regióes tropicais, segundo Roubik (1989), está relacionado com o comportamento eussocial, a perenidade da colônia e os hábitos generalizados de forrageio. Além disso, as espécies de Euglossina, que são restritas à região neotropical, e de Bombina contribuem com a abundância de Apidae sensu stricto nas regiões de menor latitude (Michener, 2000; Silveira et al., 2002).

Por outro lado, existe uma tendência de aumento na proporção de espécies de Halictidae em áreas com distúrbios (Laroca et al., 1982), enquanto que Megachilidae apresenta menor riqueza tanto no Sul do Brasil (CureHakim, 1983; Wittmann \& Hoffman, 1990), como próximo à linha do Equador (Roubik, 1989).

Analisando a distribuição das espécies entre as tribos, foi observado que Apini se destacou com 30,12\% das espécies, seguida de Megachilini $(14,46 \%)$ e Augochlorini $(10,84 \%)$ (Figura 3). Com relação aos gêneros a maior riqueza de espécies foi encontrada em Trigona $(9,64 \%)$, Megachile (8,43 $\%)$, Centris (7,23 \%), Augochloropsis, Coelioxys, Paratetrapedia e Xylocopa com 6,02 \%, cada um.

Entre as espécies coletadas, Apis mellifera foi a mais abundante $(69,79 \%$ dos indivíduos coletados). Esse resultado é semelhante ao encontrado em outros estudos realizados no Brasil (Martins, 1994; Aguiar \& Martins, 1997; Carvalho, 1999).

As espécies com comportamento social representaram $26,51 \%$ do total coletado, com $91,99 \%$ dos indivíduos. Essas espécies apresentam a capacidade de comunicar a localização das fontes de alimentos para outras operárias, além de possuírem colônias populosas (Lindauer \& Kerr, 1960), possibilitando o aparecimento nas flores de um número elevado de abelhas provenientes de uma única colônia (Sakagami et al., 1967).

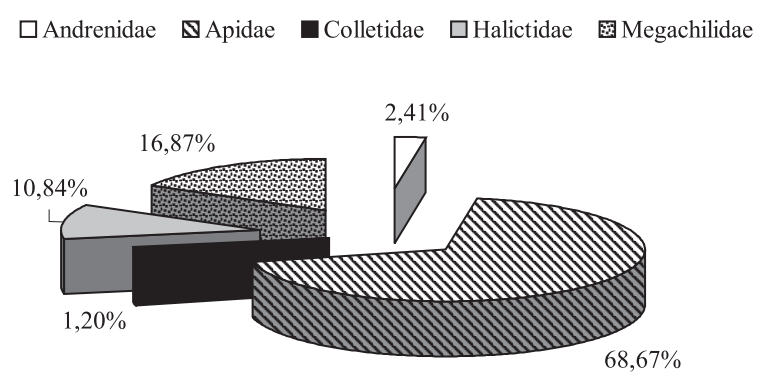

Figura 2 - Distribuição do número de espécies (\%) por família de abelhas (Hymenoptera: Apoidea) em uma área de transição cerrado-amazônia na região do Bico-do-Papagaio, Estado do Tocantins: novembro de 1999 a novembro de 2000. 


\section{ACTA \\ AMAZONICA \\ DIVERSIDADE DE ABELHAS (HYMENOPTERA: APOIDEA) \\ EM UMA ÁREA DE TRANSIÇÃO CERRADO-AMAZÔNIA}

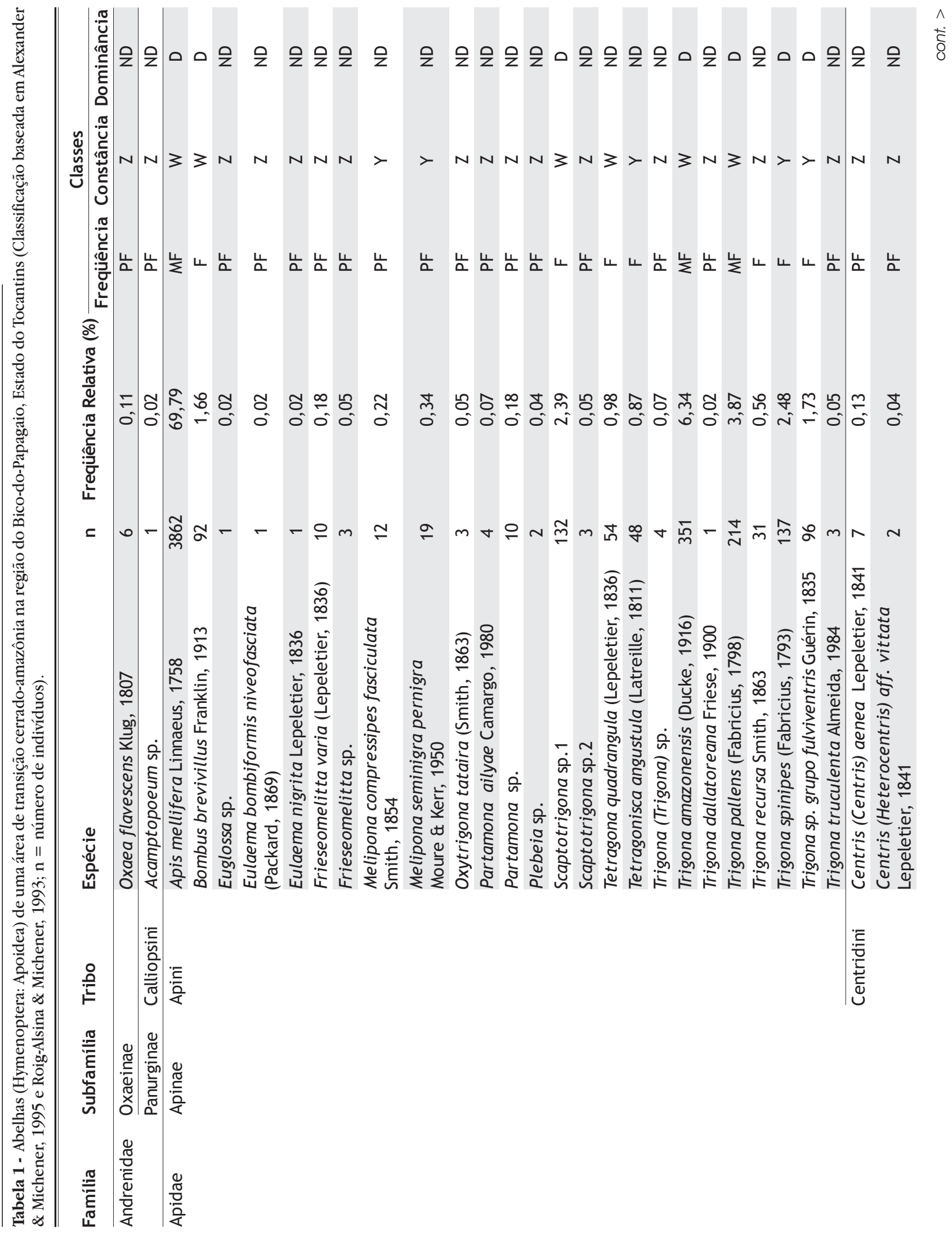




\section{ACTA \\ AMAZONICA \\ DIVERSIDADE DE ABELHAS (HYMENOPTERA: APOIDEA) \\ EM UMA ÁREA DE TRANSIÇÃO CERRADO-AMAZŌNIA}

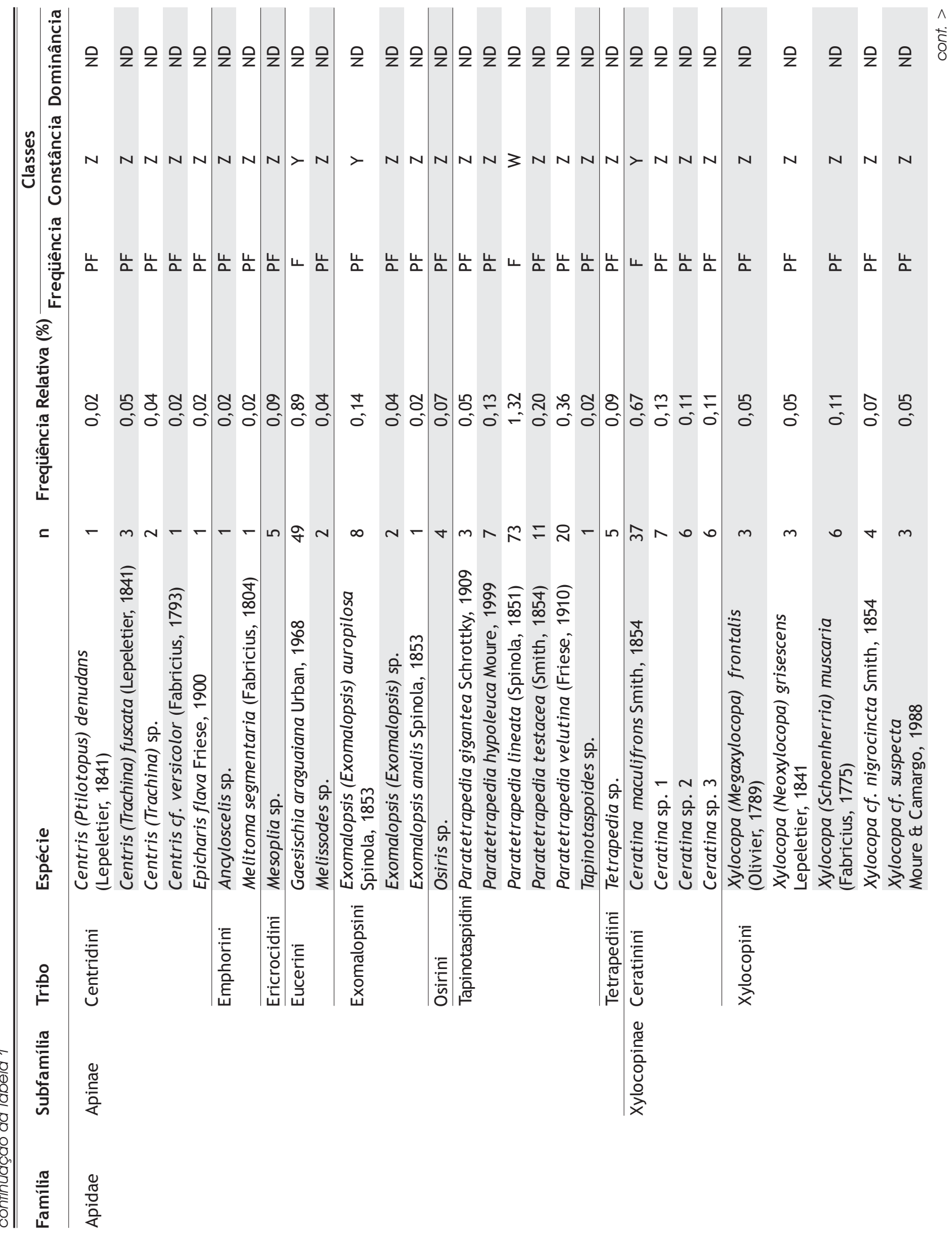




\section{ACTA \\ AMAZONICA

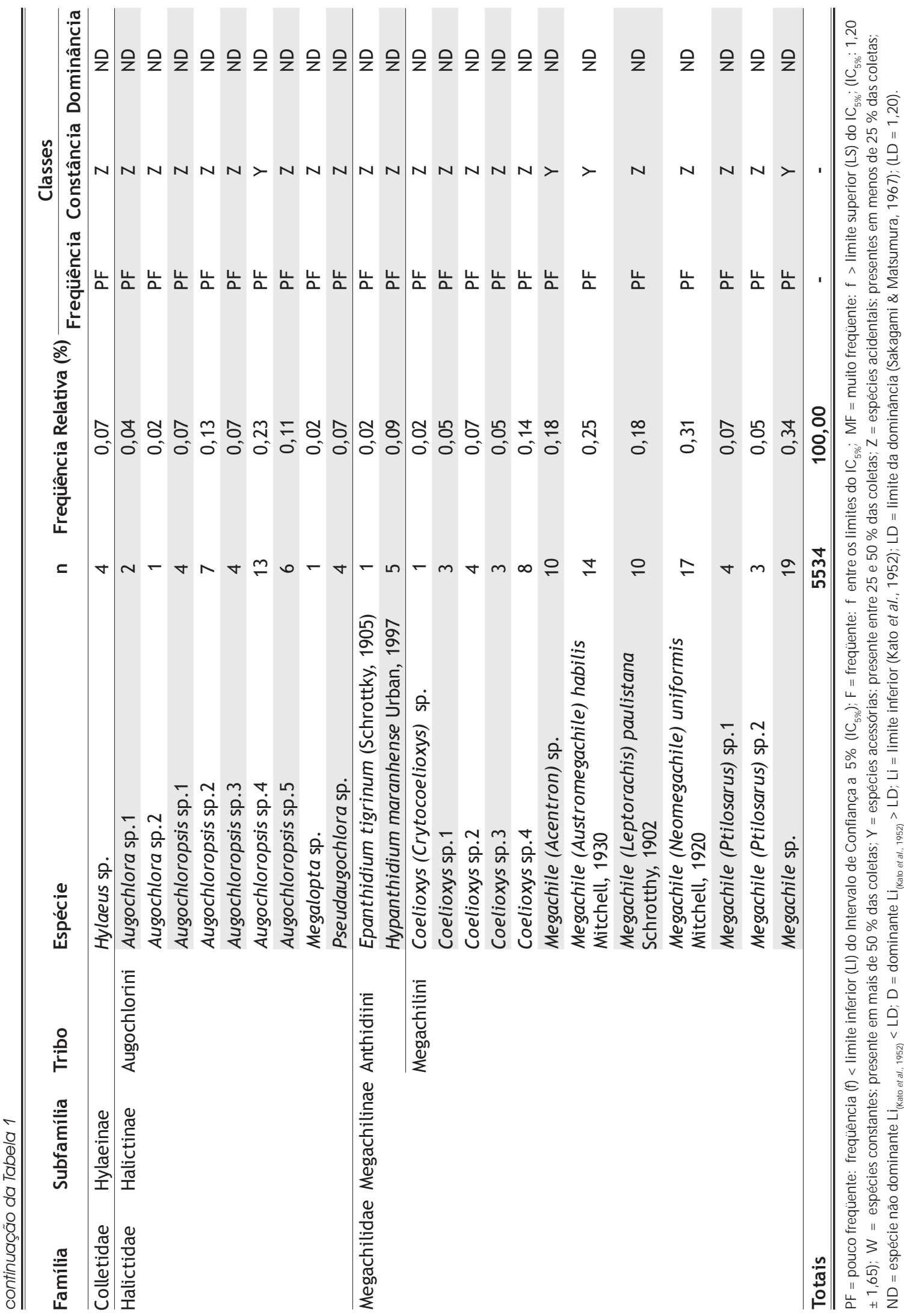




\section{ACTA AMAZONICA}

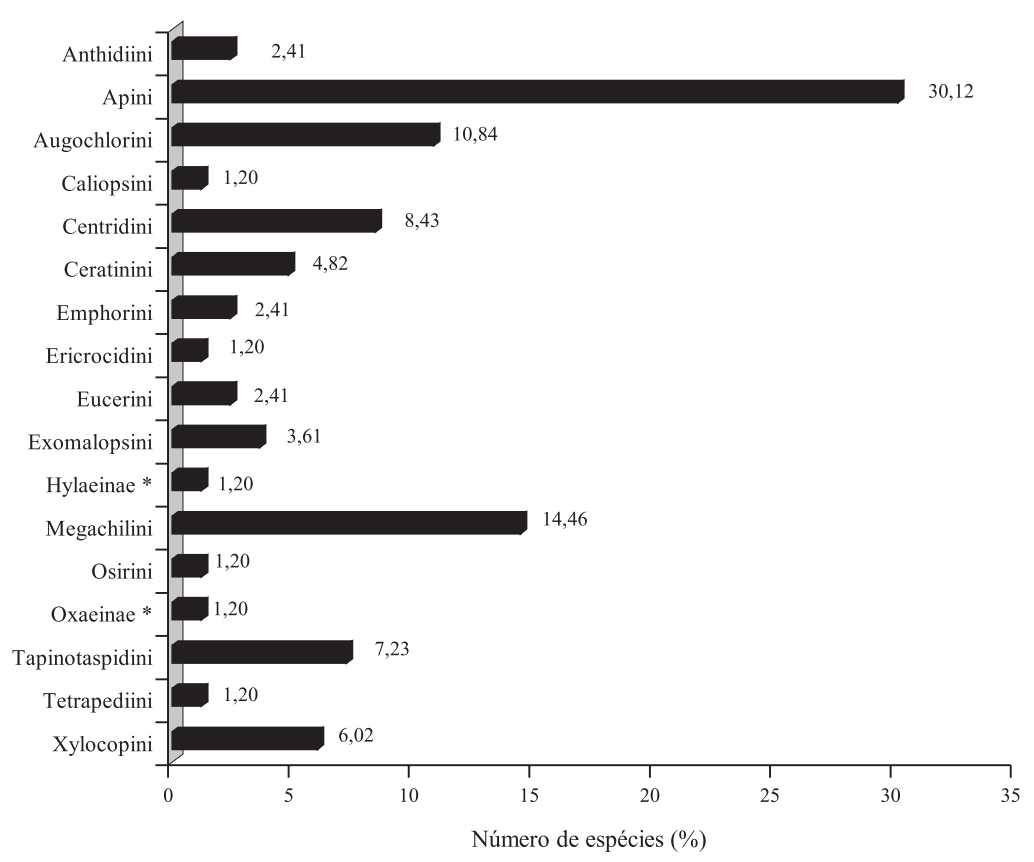

Figura 3 - Distribuição do número de espécies (\%) por tribo de abelhas (Hymenoptera: Apoidea) em uma área de transição cerrado-amazônia na região do Bico-do-Papagaio, Estado do Tocantins: novembro de 1999 a novembro de 2000. (* subfamília sem tribo definida) gênero Trigona se destacou com a maior riqueza entre essas espécies $(57,14 \%)$, sendo dominantes Trigona amazonensis, Trigona pallens, Trigona spinipes e Trigona sp. grupo fulviventris.

Considerando os maiores valores das classes freqüência $(M F)$, constância $(W)$ e dominância $(D)$ as espécies $A$. mellifera, Trigona amazonensis e Trigona pallens foram as espécies predominantes na área de estudo.

A distribuição do número de indivíduos e de espécies por coleta, baseada na Curva do Coletor, indicou que o número de coletas realizadas foi suficiente para amostrar a abundância de espécies da área estudada (Figura 4). Observa-se que ocorreu uma estabilização a partir da $21^{\text {a }}$ coleta, quando se atingiu o total de 83 espécies $(=S)$.

A comunidade de abelhas foi caracterizada por um número elevado de espécies com poucos indivíduos, enquanto que um número reduzido de espécies foi representado por muitos espécimes. Resultados semelhantes foram encontrados em diferentes habitats por Heithaus (1979), Campos (1989), Bortoli \& Laroca (1990), Barbola \& Laroca (1993). De acordo com Laroca (1992), há uma tendência de se encontrar muitas espécies com poucos indivíduos em diferentes habitats do mundo.

Os resultados obtidos neste tipo de estudo permitem apenas uma abordagem descritiva da comunidade e não devem ser considerados conclusivos, uma vez que o método de amostragem pode contribuir para a ausência de algumas espécies nas coletas (Viana, 1992).

Apesar das limitações, Sakagami et al. (1967) consideraram que a amostragem da comunidade de abelhas que visitam as flores em áreas restritas, fornece uma estimativa considerável da abundância relativa das espécies e famílias dentro da comunidade.

Os índices de abundância, diversidade e eqüitabilidade (Tabela 2 ) sugerem que a comunidade de abelhas é menos diversificada do que se esperava, uma

Tabela 2 - Índices de abundância, diversidade e eqüitabilidade da comunidade de abelhas (Hymenoptera: Apoidea) em uma área de transição cerrado-amazônia na região do Bico-do-Papagaio, Estado do Tocantins: novembro de 1999 a novembro de 2000.

\begin{tabular}{lc}
\hline \hline Índices & Valores obtidos \\
\hline$\propto$ (Margalef) & 9,51 \\
$H^{\prime}$ (Shannon-Wiener) & 1,55 \\
$\lambda$ (Simpson) & 0,49 \\
$J^{\prime}$ (Pielou) & 0,35 \\
\hline \hline
\end{tabular}




\section{ACTA \\ AMAZONICA}

DIVERSIDADE DE ABELHAS (HYMENOPTERA: APOIDEA) EM UMA ÁREA DE TRANSIÇÃO CERRADO-AMAZÔNIA

vez que a área está localização em uma zona de grande diversidade ambiental e biológica. Os valores obtidos são intermediários, quando comparados a outros habitats, como o de caatinga $(S=42)$ e o de elementos de campos ruprestes $(S=147)$ estudados por Martins (1994).
A riqueza de espécies pode ser representada graficamente pelo parâmetro $b$ da equação da reta da Figura 5. Comparando esse valor $(b=19,52)$ com os obtidos em outros estudos de comunidades de abelhas, observa-se que os resultados se aproximam mais de locais considerados

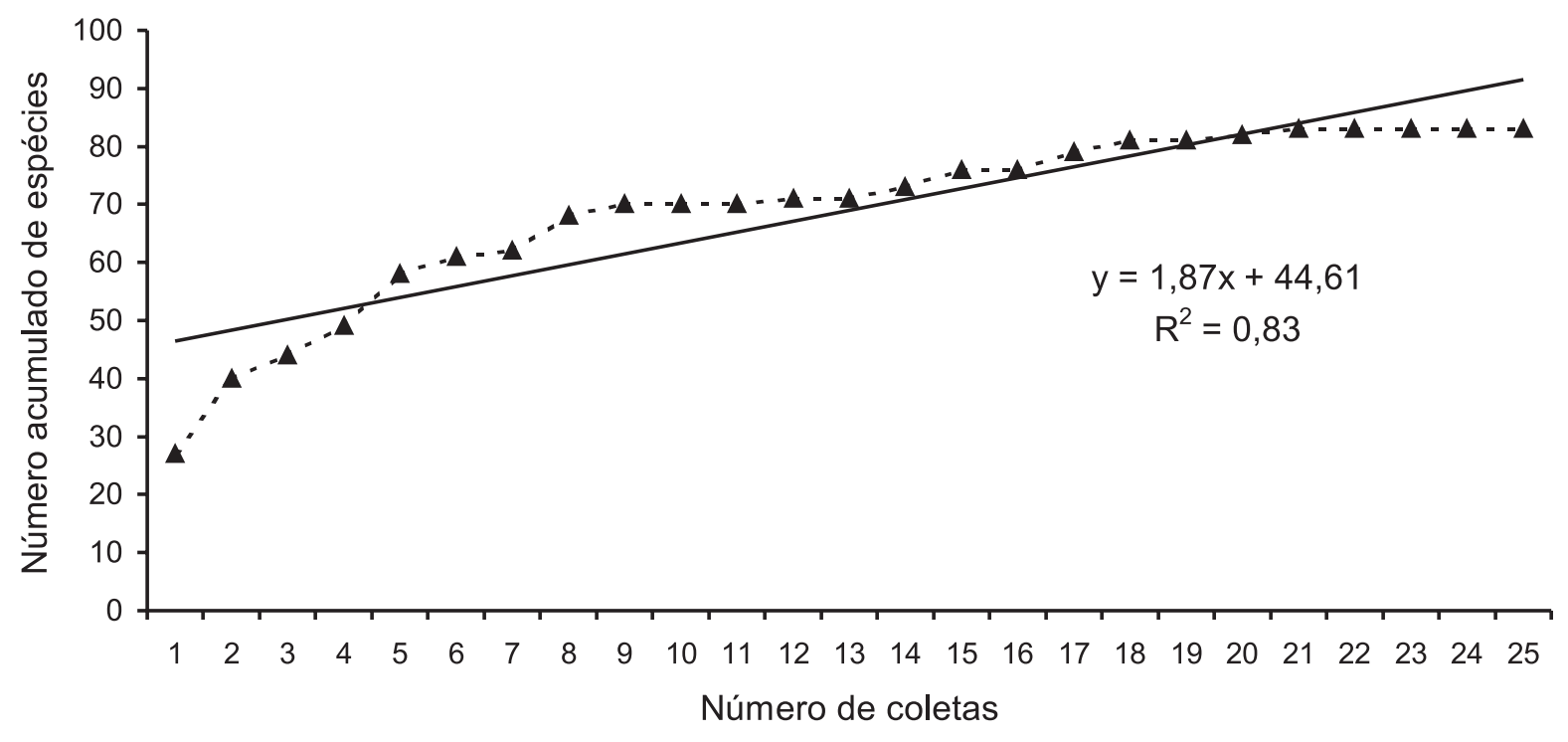

Figura 4 - Curva do Coletor para amostras de abelhas (Hymenoptera: Apoidea) coletadas em uma área de transição cerrado-amazônia na região do Bico-do-Papagaio, Estado do Tocantins: novembro de 1999 a novembro de 2000.

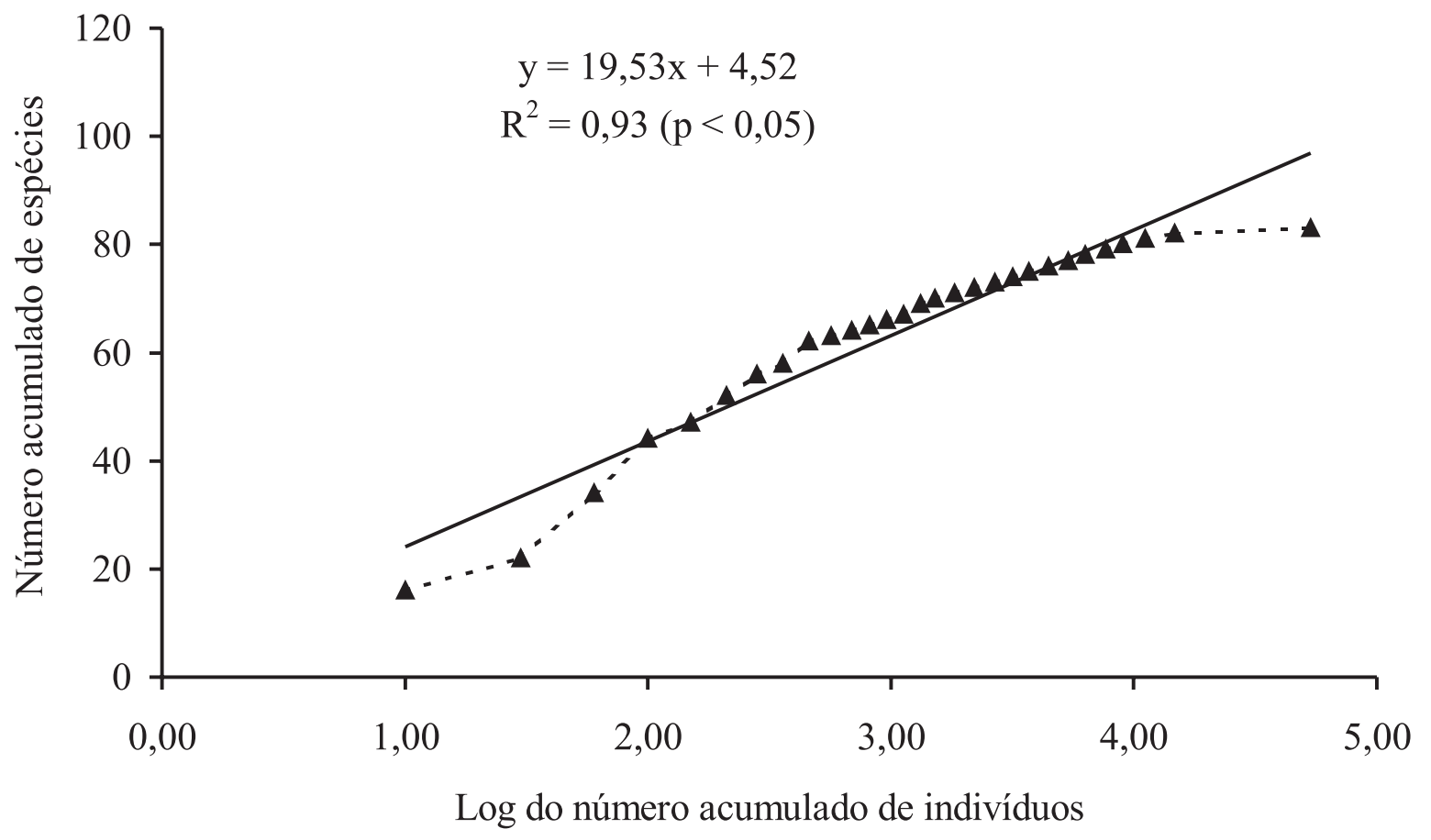

Figura 5 - Relação entre o número acumulado de indivíduos $\left(\log _{10}\right)$ e o número de espécies de abelhas (Hymenoptera: Apoidea) coletadas em uma área de transição cerrado-amazônia na região do Bico-do-Papagaio, Estado do Tocantins: novembro de 1999 a novembro de 2000. 


\section{ACTA AMAZONICA}

menos diversificados, como Casa Nova-BA $(b=15,26)$, São João do Cariri-PB $(\mathrm{b}=14,20)$ e Castro Alves-BA $(\mathrm{b}=22,67)$ (Martins, 1994; Aguiar \& Martins, 1997; Carvalho, 1999). Ambientes considerados mais diversificados apresentam valores maiores desse parâmetro como os observados em São José dos Pinhais-PR $(b=73,40)$, Lapa-PR $(b=69,90)$ e Lages-SC (b = 51,06) (Ortolan, 1989; Bortoli \& Laroca, 1990; Barbola \& Laroca, 1993; Martins, 1994).

Uma possível explicação para esse resultado é o uso intensivo da terra por atividades da agropecuária, o que tem promovido modificações profundas na fisionomia vegetal da região com implicaçóes para a comunidade das abelhas. É comum o uso de queimadas para a implantação de pastagem, comprometendo tanto as espécies de hábito solitário como as sociais. Além disso, a destruição de colônias para obtenção de mel (Meliponina) ou por causarem algum tipo de dano às culturas (Trigona spp.) é prática comum na área de estudo.

\section{AGRADECIMENTOS}

Os autores agradecem a Diretoria do RURALTINS Instituto de Desenvolvimento Rural do Estado do Tocantins e a Associação dos Apicultores do Bico-do-Papagaio - ABIPA pela iniciativa e apoio durante o PGAI/Projeto: Inventário da fauna e flora apícolas da região do Bico-do-Papagaio, Estado do Tocantins, período 1999-2001; e aos taxonomistas Dr. João Maria de Camargo (FFCLRP-USP), Dr. Eduardo A. B. Almeida (UFMG), Dr. Fernando A. Silveira (UFMG), Dr. Fernando Zanella (UFPB) e Dr. Gabriel A.R. Melo (UFPR) pela colaboração na identificação das espécies de abelhas.

\section{BIBLIOGRAFIA CITADA}

Aguiar, C.M.L.; Martins, C.F. 1997. Abundância relativa, diversidade e fenologia de abelhas (Hymenoptera, Apoidea) na caatinga, São João do Cariri, Paraíba, Brasil. Iheringia. Série Zoologia, 83: 151-163.

Aguiar, C.M.L.; Martins, C.F.; Moura, A.C. 1995. Recursos florais utilizados por abelhas (Hymenoptera, Apoidea) em área de caatinga (São João do Cariri, Paraíba). Rev. Nordest. Biol. 10(2): 101-117.

Barbola, I. F.; Laroca, S. 1993. A comunidade de Apoidea (Hymenoptera) da Reserva Passa Dois (Lapa, Paraná, Brasil): I. Diversidade, abundância relativa e atividade sazonal. Acta Biol. Paran., 22 (1/4): 91-113.

Barth, F.G. 1991. Insects and flowers - the biology of partnership. Princeton: Princeton University Press. 407p.

Batra, S.W. 1984. Solitary bees. Sci. Amer, 250: 86-93.

Bortoli, C. de, Laroca, S. 1990. Estudo biocenótico em Apoidea (Hymenoptera) de uma área restrita em São José dos Pinhais (PR, Sul do Brasil), com notas comparativas. Dusenia, 15: 1-112.

Campos, M. J. O. 1989. Estudo das interações entre a comunidade de Apoidea, na procura de recursos alimentares e a vegetação de cerrado na reserva de Corumbatai-SP. Tese de Doutorado, Universidade Federal de São Carlos. São Carlos, 114p.
Carvalho, C.A.L. de. 1999. Diversidade de abelhas (Hymenoptera, Apoidea) no município de Castro Alves-BA. Tese de Doutorado, Universidade de São Paulo, Piracicaba, 104p.

Colwell, R.K.; Coddington, J.A. 1994. Estimating terrestrial biodiversity through extrapolation. Philosophical Transactions Royal Society of London. B., 345: 101-118.

Cure, J. R.; Bastos Filho, G. S.; Oliveira, M. J. F. de; Silveira, F. A. da. 1993. Levantamento de abelhas silvestres na zona da mata de Minas Gerais. I- Pastagem na Região de Viçosa (Hymenoptera, Apoidea). Rev. Ceres, 40(228): 130-161.

Cure-Hakim, J. R. 1983. Estudo ecológico da comunidade de abelhas silvestres (Hymenoptera, Apoidea) do Parque da Cidade, comparado ao de outras áreas de Curitiba, Paraná. Dissertação de Mestrado, Universidade Federal do Paraná. Curitiba, 100p.

Frankie, G. W., Haber, W.W.; Opler, P. A.; Bawa, K.S. 1983. Characteristics and organization of the large bee pollination system in the Costa Rica dry forest. In.; C. E. Jones \& R. J. Little (eds.), Handbook of experimental pollination biology. New York, Scientific and Academic Editions, $558 \mathrm{p}$

Heithaus, E.R. 1979.Community structure of neotropical flower visiting bees and wasps: diversity and phenology. Ecology, 60 (1):190-202

Kato, M., Matsuda, T., Yamashhita, Z. 1952. Associative ecology of insects found in paddy field cultivated by various planting forms. Sci. Rep. Toboku Univ. 19: 291-301.

Kevan, P.G.; Baker, H.G. 1983. Insects as flower visitors and pollinators. Ann. Rev. Ent., 28: 407-53.

Laroca, S. 1992. Community ecology in bees: relative importance of rare and common species in some holartic and neotropical sites. Rev. Bras. de Zool., 9 (1/2): 131-137.

Laroca, S.; Cure, J. R.; Bortoli, C. de. 1982. A associação de abelhas silvestres (Hymenoptera, Apoidea) de uma área restrita no interior da cidade de Curitiba (Brasil): uma abordagem biocenótica. Dusenia, 17(3): 93-117.

Lindauer, M.; Kerr, W.E. 1960. Communication between the workers of stingless bees. Bee World, 41: 29-41.

Ludwig, J.A.; Reynolds, J.S. 1988. Statistical ecology-a priner on methods and computing. New York, John Wiley \& Sons, 337p.

Martins, C.F. 1994. Comunidade de abelhas (Hym., Apoidea) da caatinga e do cerrado com elementos de campo rupestre do Estado da Bahia, Brasil. Rev. Nordest. Biol., 9(2): 225-257.

Matheson, A.; Buchmann, S.L.; O'toole, C.; Westrich, P.; Williams, I.H. 1996. The conservation of bees. London, Academic Press, 254p.

Michener, C.D. 2000. The bees of the world. Baltimore, The Jonhs Hopkins University Press, 913p.

Ortolan, S.M.L.S. 1989. Biocenótica de Apoidea (Hymenoptera) de áreas de cultivo de macieira (Pyrus malus) em Lages Santa Catarina, com notas comparativas e experimento preliminar de polinização com Plebeia emerina. Dissertação de Mestrado, Universidade Federal do Paraná, Curitiba, 170p.

Pielou, E.C. 1977. Mathematical ecology. New York: John Wiley. 385p.

Proctor, M., Yeo, P., Lack, A.. 1996. The natural bistory of pollination. London, Harper Collins Publishers, 479p. 
Roig-Alsina, A.; Michener, C.D. 1993. Studies of the phylogeny and classification of long-tongued bees (Hymenoptera: Apoidea). Univ. Kansas Sci. Bul., 55 (4/5): 123-173.

Roubik, D.W. 1989. Ecology and natural history of tropical bees. Cambridge: Cambridge University Press, 514p.

Roubik, D.W. 1993. Tropical pollinators in the canopy and understory: field data and theory for stratum preferences. $J$. Ins. Behav. 6: 659-73.

Sakagami, S.F., Laroca, S., Moure, J.S.1967. Wild bees biocenotics in São José dos Pinhais (Pr), South Brazil - preliminary report. J. Fas. Sci Hokkaido Univ. Ser. 6, Zoology, 19: 253-91.

Sakagami, S.F.; Matsumura, T. 1967. Relative abundance, phenology and flower preference of andrenid bees in Sapporo, north Japan (Hymenoptera, Apoidea) Jap. Journal. Ecol., 17 (6): 237-50.

Silveira, F.A.; Campos, M.J.O. 1995. A melissofauna de Corumbataí (SP) e Paraopeba (MG) e uma análise da biogeografia das abelhas do cerrado Brasileiro (Hymenoptera, Apoidae). Rev. Bras. Ent., 39 (2): 371-401.
Silveira, F.A.; Melo, G.A.R.; Almeida, E.A.B. 2002. Abelhas brasileiras - sistemática e identificação. Belo Horizonte, Fundação Araucária, 253p.

Silveira Neto, S., Nakano, O., Vila Nova, N.A. 1976. Manual de ecologia dos insetos. Piracicaba, Ceres, $419 \mathrm{p}$.

Viana, B. F. 1992. Estudo da composição da fauna de Apidae e da flora apícola da Chapada Diamantina, Lençóis - Babia $\left(12^{\circ} 34^{\prime} S ; 41^{\circ} 23^{\prime} W\right)$. Dissertação de Mestrado, Instituto de Biociências, Universidade de São Paulo, São Paulo, 140p.

Wittmann, D.; Hoffman, M. 1990. Bees of Rio Grande do Sul, Southern Brazil (Insecta, Hymenoptera, Apoidea). Iheringia. Série Zoologia, 70: 17-43.

\section{RECEBIDO EM 11/08/2003 \\ ACEITO EM 15/03/2004}

